\title{
Electroencephalographic Recordings in the Canine: Effects of Low Dose Medetomidine or Dexmedetomidine Followed by Atipamezole*
}

\author{
Laura C. Tepper ${ }^{1}$, Andy Shores ${ }^{2,3 \#}$ \\ ${ }^{1}$ Bay Street Animal Hospital, Staten Island, NY, USA \\ ${ }^{2}$ Veterinary Specialty Center, Starkville, MS, USA \\ ${ }^{3}$ College of Veterinary Medicine Mississippi State University, Mississippi State, MS, USA \\ Email: lctepper@gmail.com, ${ }^{\#}$ shores@cvm.msstate.edu
}

Received November 24, 2013; revised December 24, 2013; accepted December 31, 2013

Copyright (c) 2014 Laura C. Tepper, Andy Shores. This is an open access article distributed under the Creative Commons Attribution License, which permits unrestricted use, distribution, and reproduction in any medium, provided the original work is properly cited. In accordance of the Creative Commons Attribution License all Copyrights (C) 2014 are reserved for SCIRP and the owner of the intellectual property Laura C. Tepper, Andy Shores. All Copyright (c) 2014 are guarded by law and by SCIRP as a guardian.

\section{ABSTRACT}

Objectives: 1) To describe electroencephalogram (EEG) appearance in the awake dog and compare these results with EEG recordings after low dose medetomidine (2 $\mu \mathrm{g} / \mathrm{kg}$ IV) followed by atipamezole (10 $\mu \mathrm{g} / \mathrm{kg}, \mathrm{IM}) ; 2)$ To institute EEG recordings after low dose medetomidine or dexmedetomidine as a standard of practice if focal abnormalities and amplitudes were not significantly altered by pharmaceuticals in Phase 1 of this study. Methods: Electroencephalograms were performed on eight clinical canine patients with suspected intracranial disease involving the prosencephalon. A five lead montage was used to record the EEGs. Each dog had an awake, baseline recording followed by an EEG performed after administration of low dose medetomidine $(2 \mu \mathrm{g} / \mathrm{kg} \mathrm{IV})$ then atipamezole $(10 \mu \mathrm{g} / \mathrm{kg}$, IM). In the second phase of this study, the same dose of medetomidine or dexmedetomidine at $1 \mu \mathrm{g} / \mathrm{kg}$ IV and atipamezole $(10 \mu \mathrm{g} / \mathrm{kg}$, IM) were used in the evaluation of 20 clinical patients with suspected neurologic disease. Results: In Phase 1, awake recordings were laced with movement artifacts. After medetomidine and atipamezole, EEG waveforms were slower. Following atipamezole, however, the frequencies were observed to increase with time. Statistical evaluation revealed significantly more artifacts in baseline recordings. No statistically significant change was observed in focal abnormalities or amplitude. In Phase 2 , the $\alpha 2$-adrenoreceptor agonists followed by atipamezole without the use of lidocaine produced clinically reliable results. Clinical Significance: Quality and diagnostic electroencephalogram (EEG) recordings are frequently inconvenient to obtain in the awake dog. Movement results in artifacts and dislodged leads. Administration of low dose medetomidine or dexmedetomidine followed by atipamezole reliably reduced the impact of movement artifacts and produced clinically valid EEG recordings in dogs.

\section{KEYWORDS}

Dog; Brain; Electroencephalogram; Medetomidine; Atipamezole; Dexmedetomidine

\section{Introduction}

Electroencephalogram (EEG) recordings in dogs are fre-

\footnotetext{
*All work was done at Mississippi State University, College of Veterinary Medicine. No grant or other financial support was obtained. Presentation: Abstract of portions of this paper was presented as a poster on June 6-9, 2007 at the ACVIM conference in Seattle, WA. At the time of presentation, the title was, "The use of medetomidine and atipamezole for electroencephalographic recordings in dogs."

"Corresponding author.
}

quently performed without the administration of anesthetics or sedatives. Awake canine patients sometimes do not cooperate during all or parts of the EEG recording, resulting in numerous artifacts and dislodged leads, which interfere in obtaining high quality and diagnostic tracings. In some patients, placement of subcutaneous electrodes or the act of physical restraint is excessively stressful. Anesthetic or sedative drugs are useful in reducing patient stress, pain and movement; however, to 
date, use of these drugs has interfered with EEG tracings and interpretation [1-3]. Medetomidine contains the dextro and levo components of the drug and is an $\alpha 2$-adrenoreceptor agonist with sedative and analgesic properties that are dose dependant in duration and depth. The dextro form is the active form. Dexmedetomidine contains only the dextro (active form) and is the currently marketed pharmaceutical for veterinary use. Medetomidine's sedative activity is generally attributed to its stimulation of the $\alpha-_{2}$ adrenoceptors in the locus coeruleus, thereby inhibiting the ascending activation projection to the forebrain and reducing sensitivity to environmental stimuli [4].

Since medetomidine is thought to work primarily at the level of the locus coeruleus [4], our hypothesis was, medetomidine should not significantly change the overall trace quality if used at a low dose intravenously. And, since dexmedetomidine is the active form of the drug, use of this at one-half the dose would have similar results. Atipamezole competitively inhibits alpha2-adrenergic receptors and is used clinically in the dog to reverse the effects of medetomidine; theoretically, any changes caused by medetomidine should be reversed by the atipamezole.

The EEG records cerebral cortical neuron activity. Cerebral cortical neurons are influenced by the ascending reticular activating system of the brainstem. The EEG waveform varies with levels of consciousness. The alert state is characterized as low voltage fast wave activity. High voltage slow wave activity is observed with drowsiness, non-REM sleep, or certain pathologic states. Sedatives, tranquilizers and anesthetics produce high voltage slow wave activity similar to slow wave sleep patterns. REM sleep produces an EEG pattern similar to the awake state $[1,5]$.

This study was designed to compare the cortical activity of canine patients with suspected intracranial disease when awake with cortical activity following sedation with low dose intravenous medetomidine, followed by cortical activity after administration of intramuscular atipamezole to reverse the medetomidine.

\section{Materials and Methods}

In Phase 1, eight canine patients referred to Mississippi State University, College of Veterinary Medicine had EEGs recorded as part of the investigation for potential intracranial disease involving the prosencephalon. Some patients were currently on anticonvulsant therapy. Each patient had a complete physical and neurologic examination. All patients selected were well hydrated, had a good body condition score, and had a normal heart rate, respiratory rate, body temperature and blood pressure. No significant abnormalities on CBC, serum chemistry or urinalysis were noted. The electrodes used were $12 \times 4$ mm, $0.5 \times 27$ G disposable subdermal EEG needles, made of stainless steel, coated with polytetrafluoroethylene (Neuroline ${ }^{\circledR}$ disposable subdermal needle electrodes; Ambu). The electrodes were placed using a modified Redding montage [6]. The electrodes were placed subcutaneously at the right and left frontal positions (F4 and F3 respectively), right and left parietal positions (P4 and P3 respectively), the center position (CZ), and a ground electrode behind the neck (Figure 1). Leads were tested initially to determine all had low electrical impedance and no muscle interference.

An injection of intramuscular 2\% lidocaine was used locally at the electrode sites if necessary to decrease muscle interference. The dose of $2 \%$ lidocaine used was approximately $2 \mathrm{mg}(0.1 \mathrm{ml})$ blebs at each needle insertion, without exceeding $2 \mathrm{mg} / \mathrm{kg}$ total dose [7]. Each recording was performed in the same manner, in a low lit, quiet room, with the patient gently restrained in sternal recumbency. Each EEG recording was divided into three ten minute sections: baseline, post-medetomidine and post-atipamezole; for statistical purposes, each ten minute section was referred to as time 1 , time 2 and time 3 respectively. An awake, baseline EEG recording was performed for approximately ten minutes. The same recording technique was repeated after administration of medetomidine ( $2 \mu \mathrm{g} / \mathrm{kg}, \mathrm{IV}$ ) and after reversal with atipamezole (10 $\mu \mathrm{g} / \mathrm{kg}$, IM). The data was collected and stored on a computer program (Easy EEG version 1.7, Cadwell Laboratories). Each tracing was evaluated and data was extracted including: the percentage of the tracing affected by artifacts, number of focal abnormalities (spikes and spindles) per tracing, average $\mu \mathrm{V}$, and average $\mathrm{Hz}$. Heart rate, pulse and respiratory rate were monitored from the time of medetomidine injection until re-

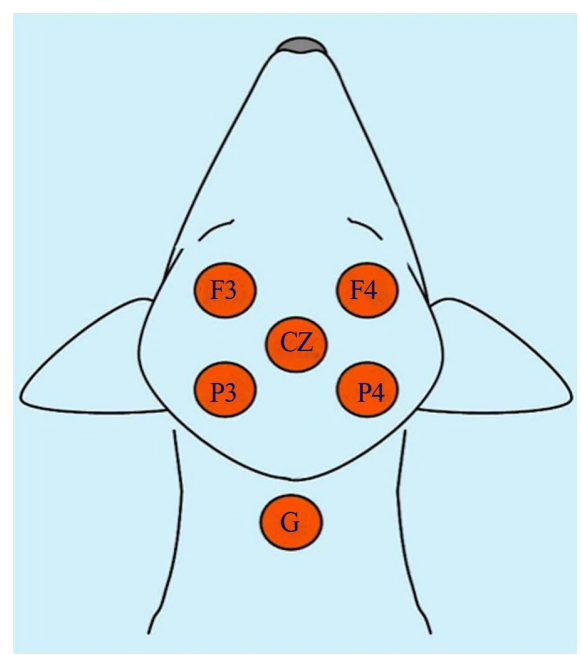

Figure 1. Five-lead electroencephalogram (EEG) electrode placement protocol used on the canine patients in this study. Even numbers $=$ right side, odd numbers $=$ left side, $\mathrm{z}=$ midline, $\mathrm{F}=$ frontal, $\mathrm{P}=$ parietal, $\mathrm{C}=$ central, $\mathrm{G}=$ ground. 
turning to normal (approximately 10 minutes after the atipamezole injection).

Statistical evaluation was then performed using the Mixed procedure (SAS for Windows 9.1, SAS Institute Inc., Cary, NC, USA) accounting for repeated measures of dogs over time (3 ewp value of $<0.05$ ). Response variables included percentage of the tracing affected by artifacts, number of focal abnormalities (spikes and spindles) per tracing, average $\mu \mathrm{V}$, and average $\mathrm{Hz}$. For each response variable, a model incorporating fixed effects for treatment, and random effects for dog was fit. The treatment variable included three time periods, namely baseline (non-drug), following medetomidine, and then following atipamezole portions of the EEG recordings. An unstructured covariance structure was used to model variation within dogs measured over three time periods.

The second phase of this study instituted the use of medetomidine (2 $\mu \mathrm{g} / \mathrm{kg}$ IV) or dexmedetomidine (1 $\mu \mathrm{g} / \mathrm{kg}$ IV) then atipamezole $(10 \mu \mathrm{g} / \mathrm{kg}$, IM) in clinical patients without the use of the lidocaine injections as the standard of practice for obtaining EEG recordings. Use of the dexmedetomidine began after the manufacturer discontinued marketing medetomidine for the veterinary market.

\section{Results}

Eight dogs (mean age, 4.6 years; range 11 months to 9 years) were entered into the first phase of the study: 5 females (62.5 percent; one intact) and 3 males (37.5 percent; 2 intact). Breeds included mixed (2), Boston terrier (1), Shih Tzu (1), Miniature Pinscher (1), Dachshund (1), Golden Retriever (1), and French bulldog (1). Presenting signs included seizures (5 dogs, 62.5 percent), general proprioceptive deficits (6 dogs, 75 percent), circling (3 dogs, 37.5 percent), unilateral blindness ( 2 dogs, 25 percent), unilateral nasal hypalgesia (3 dogs, 37.5 percent), neck pain ( 1 dog, 12.5 percent), and nystagmus ( 3 dogs, 37.5 percent). Medications that the patients were receiving prior to the EEG included phenobarbital (4 dogs, 50 percent), doxycycline (3 dogs, 37.5 percent), and potassium bromide (1 dog, 12.5 percent). Electroencephalographic recordings showed various activities such as spikes and spindles. EEG findings in each patient during each 10 minute segment (baseline, medetomidine and atipamezole) were recorded and summarized (Table 1). The traces were initially analyzed by subjective visual assessment. The awake recordings were laced with movement and muscle artifacts (Figures 2(a) and 3(a)). Some portions of the recordings without the use of sedatives were repeated because of lead displacement. After medetomidine administration, the EEG waveforms were slightly slower (Figures 2(b) and 3(b)). The same was true initially following atipamezole administration (2C and $3 \mathrm{C}$ ); however, the waveform frequencies were ob-
Table 1. EEG results during each 10 minute segment: baseline, medetomidine and atipamezole (time 1, time 2 and time 3 respectively). Percent artifact $=$ the percentage of the tracing affected by artifacts. Focal abn $=$ Number of focal abnormalities (spikes and spindles). Avg $\mu \mathrm{V}$ and avg $\mathrm{Hz}=$ the average $\mu \mathrm{V}$ and $\mathrm{Hz}$ in each ten minute sequence. Values in same column with the same letter notation (A, B, or $\mathrm{C}$ ) are not statistically different $(P>0.05)$.

\begin{tabular}{cccc}
\hline & \% Artifacts & Focal Abnormalities & Average $\boldsymbol{\mu V}$ \\
\hline Baseline & $78.71(\mathrm{~A})$ & $0.13(\mathrm{~A})$ & $15.94(\mathrm{~A})$ \\
Medetomidine & $18.99(\mathrm{~B})$ & $4.25(\mathrm{~A})$ & $33.69(\mathrm{~A})$ \\
Atipamezole & $5.67(\mathrm{C})$ & $5.63(\mathrm{~A})$ & $31.44(\mathrm{~A})$ \\
Average Hz & $30.94(\mathrm{~A})$ & $17.5(\mathrm{~B})$ & $16.5(\mathrm{~B})$ \\
\hline
\end{tabular}

served to gradually increase with time, becoming closer to that of the awake animal.

Statistical analysis revealed significantly more artifacts in the baseline recordings than in the medetomidine and atipamezole recordings. There was a significantly slower wave pattern $(\downarrow \mathrm{Hz})$ after medetomidine and following atipamezole administration. No statistically significant change was observed in the focal abnormalities or the amplitude of the tracings before and after administration of each of the drugs. Overall, focal abnormalities were observed more frequently after medetomidine and after atipamezole; however, this was only significant at a $\mathrm{P}$ value of $<0.10$.

After the administration of medetomidine, mild to moderate bradycardia and bradypnea were noted in all of the patients. These side effects gradually diminished and returned to normal approximately 10 - 20 minutes after the intramuscular injection of atipamezole. All dogs appeared physically healthy throughout the study.

Further neurologic work up such as CT or MRI of the brain and/or CSF analysis was performed in most of the patients to determine the cause of the neurologic signs. The EEG results correlated with these findings.

In the second phase of the study, after reviewing the results of the first phase, results of the EEG recordings were made using only medetomidine (8 dogs) or dexmedetomidine (12 dogs) followed by atipamezole in 20 patients. None of the patients required restraint for needle placement and muscle artifacts were minimal to absent. Sixteen of the dogs had focal abnormalities or changes in amplitude and 4 dogs had normal EEG recordings. These findings correlated with the additional clinical evaluations and diagnostic results.

\section{Discussion}

This article describes the typical EEG procedure and suggests the use of low dose medetomidine followed by atipamezole in select cases where a quality EEG recording is difficult to obtain due to the behavior and/or movement of the animal. 


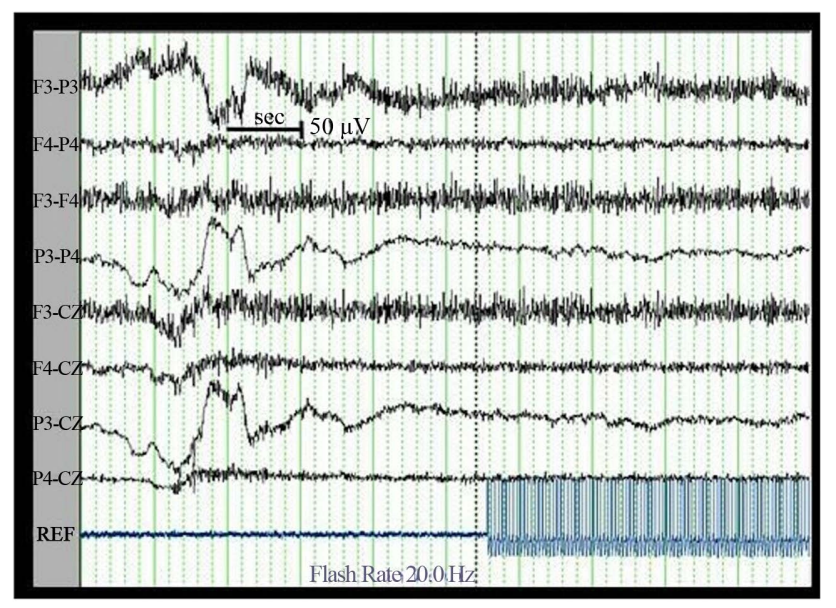

(a)

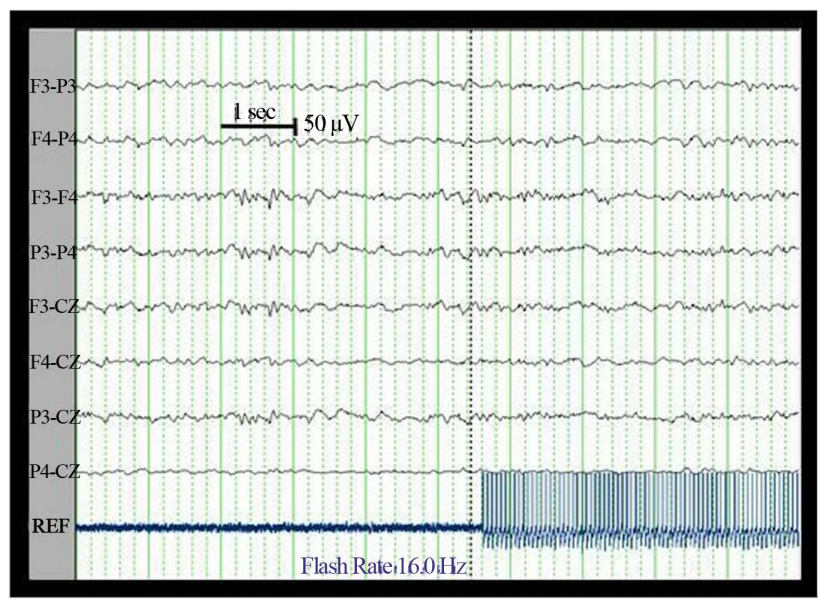

(b)

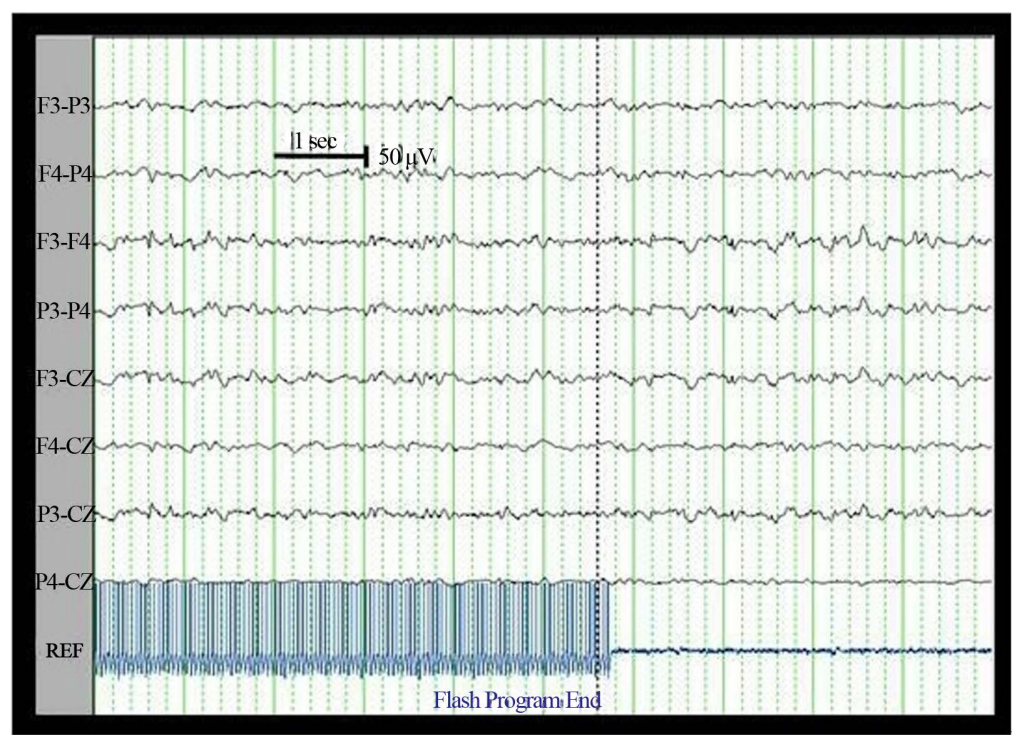

(c)

Figure 2. (a) Five lead EEG of a 2-year old spayed female Golden Retriever with seizures. Medications included phenobarbital and potassium bromide. Note the muscle and movement artifacts making this EEG difficult to interpret. (b) EEG of the same dog in Figure 2(a) after administration of medetomidine ( $2 \mu \mathrm{g} / \mathrm{kg}$, IV). There is marked improvement in the quality of the tracings. The tracings reveal low voltage fast wave activity at $3-5 \mu \mathrm{V}$ and $35-45 \mathrm{~Hz}$ with a generalized underlying pattern of high voltage slow wave activity at $20-25 \mu \mathrm{V}$ and $5-10 \mathrm{~Hz}$. There are bursts of slow wave activity from the left hemisphere. This is an abnormal EEG change consistent with a left sided cerebral abnormality of unknown origin. (c) EEG of the same dog in Figure 2(a) and (b) after administration of atipamezole (10 $\mathrm{\mu g} / \mathrm{kg}, \mathrm{IM})$. Low voltage slow wave activity at 3 - 5 $\mu \mathrm{V}$ and 35 - $45 \mathrm{~Hz}$ with overriding high voltage slow wave activity. The slow wave activity is most prominent in leads associated with the left hemisphere. There are occasional spikes associated with the left frontal lead. These EEG changes are also consistent with a left sided cerebral abnormality. REF = Reference Lead showing ECG, Photic Stimulation events, or Marks for Other Noted Activity occurring during the recording.

In the baseline recordings, animal movement, poor electrode contact, and electrode movement caused interference. A good quality electroencephalographic recording was obtained after the use of low dose intravenous medetomidine followed by intramuscular atipamezole.

Electroencephalography (EEG) records spontaneous electrical activity of the cerebral cortex and it is used in veterinary practice to help diagnose and monitor cerebral diseases [1]. Currently, some institutions are using the
EEG test less frequently with the increased availability of MRI and CT. However, EEG is still useful in patients with forebrain disease and may be the only diagnostic test affordable to some owners. Although it does not provide a specific diagnosis, the EEG can be used as a tool to determine the presence of cerebral disease and if it is focal, diffuse, acute, chronic, inflammatory or degenerative [1,5]. It is also a helpful diagnostic device to monitor response of treatment in certain intracranial dis- 


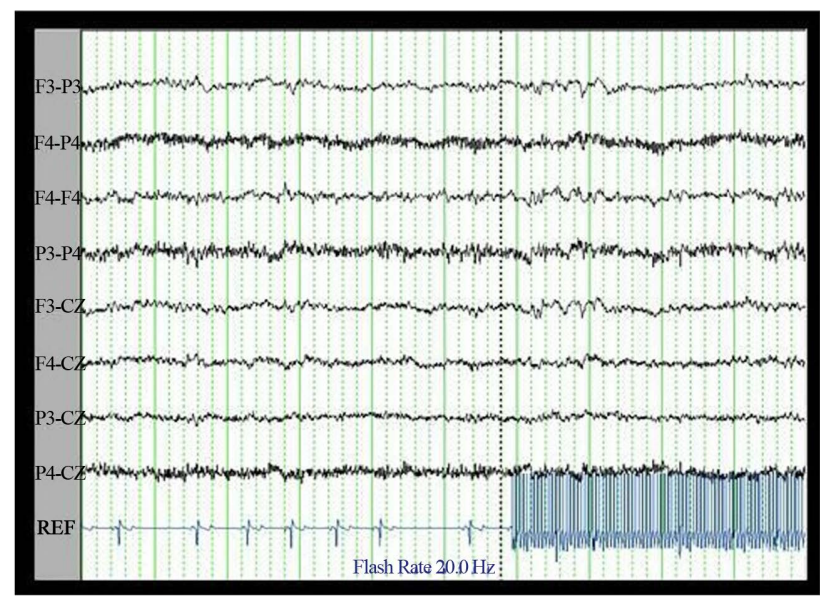

(a)

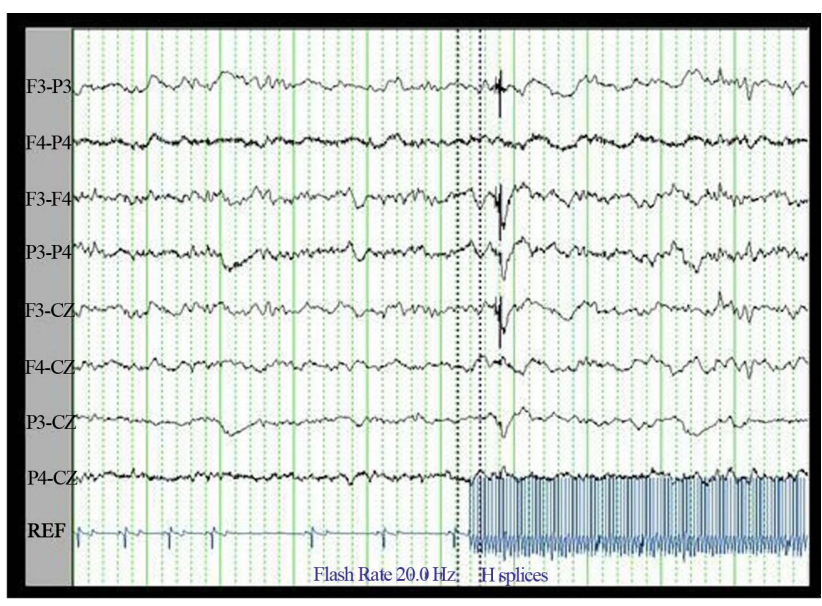

(b)

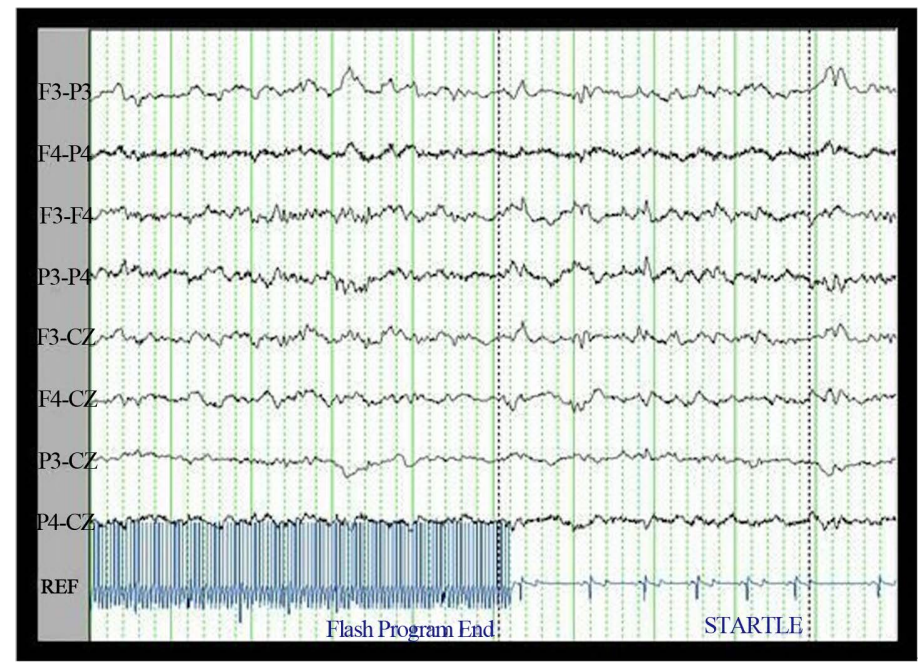

(c)

Figure 3. (a) Five lead EEG of a 7 year old intact male French bulldog with seizures, circling to the left, nasal hypalgesia on the right, general proprioceptive deficits on the right side and blind in the right eye. The patient was administered maintenance phenobarbital. This EEG shows low voltage fast wave activity at $10-15 \mu \mathrm{V}$ and $35-40 \mathrm{~Hz}$ with bursts of slow wave activity originating from the F3 lead. There are significant movement artifacts. This is an abnormal EEG consistent with a left frontal focal abnormality. (b) EEG of the same dog in Figure 3(a) after administration of medetomidine (2 $\mathrm{kg} / \mathrm{kg} \mathrm{IV).}$ Low voltage fast wave activity is present at $3-5 \mu \mathrm{V}$ and $30-35 \mathrm{~Hz}$ with an overriding pattern in the F3 lead of high voltage slow wave activity at $5-15 \mathrm{~Hz}$ and $25-40 \mu \mathrm{V}$. Spikes are present, originating from the left frontal lobe. These EEG changes are consistent with a left frontal lesion. (c) EEG of the same dog in Figure 3(a) and (b) after administration of atipamezole (10 $\mu \mathrm{g} / \mathrm{kg}, \mathrm{IM})$. High voltage slow wave activity at $20-60 \mathrm{~Hz}$ and $4-15 \mathrm{~Hz}$ with overriding low voltage fast wave activity at 5 - 10 $\mu \mathrm{V}$ and $25-30 \mathrm{~Hz}$. There is a slower wave pattern associated mostly with the left side and F3 spikes. These EEG changes are also consistent with a left frontal lesion. A CT of the brain revealed a mass dorsal to and surrounding the left petrous temporal bone. Based on history and CT appearance, the presumptive diagnosis was a meningioma. REF = Reference Lead showing ECG, Photic Stimulation events, or Marks for Other Noted Activity occurring during the recording.

eases. Some examples of diseases that EEG could be useful to either diagnose and/or monitor treatment include seizure disorders, encephalitis, hydrocephalus, forebrain mass, and to help rule-in or rule-out intracranial disease in patients with nonspecific signs of mental dullness, mentation change or episodes.

Alpha-2 agonist's sedative activity is mainly thought to be related to the hyperpolarization of the $\alpha_{2}$ adrenoceptors in the locus coeruleus [4]. Clonidine, a central- ly-acting $\alpha_{2}$ agonist produces slow wave sleep in dogs [8]. Another study looking at medetomidine's effects on EEG's found their tracings were also similar to slow wave sleep [9]. Our findings also show a slower wave EEG pattern after administration of an $\alpha_{2}$ agonist. Medetomidine's sedative effects are thought to be dose related [10] and therefore, may explain the mild decrease in wave pattern observed at the low dose ( $2 \mu \mathrm{g} / \mathrm{kg}, \mathrm{IV})$ used in our study. 
Electroencephalogram (EEG) characterization with the use of medetomidine as a single drug has been described before, however, its use was purely intramuscularly at doses 10 - 80 times the dose used in this study [9]. The patients evaluated in our study were lightly sedated, in a quiet room. We did not feel a need for the patients to be fully sedate, as we were not performing tasks that would require pain control.

In this study, after medetomidine administration, the dogs were less arousable which made the placement of the electrodes easier; this reduced the amount of muscle artifact as well. Since the initial phase of this study, use of dexmedetomidine or medetomidine followed by atipamezole has produced quality, clinically relevant EEG recordings.

Alpha-2 adrenoceptor stimulation has been thought to delay kindling, and in rats, atipamezole was shown to lower daily seizure frequency [11]. These findings suggest a decreased chance of inducing seizure activity after atipamezole administration.

Alpha-2 agonists inhibit sympathetic tone leading to side effects of hypotension, bradycardia, sedation, analgesia and hypothermia. Medetomidine inhibits neuron firing and release of transmitters, involved in regulation of sympathetic function, cardiovascular and endocrine function, cognition and nociception. It inhibits renin release and causes vasoconstriction by contraction of vascular smooth muscle. Atipamezole reverses the sedative and physiologic changes in patients that have received alpha 2 agonists. There is a more profound effect after intravenous administration. Systemic blood pressure initially increases as a response to the bradycardia; however it gradually lowers with time $[10,12,13]$. Sedation is most effective in quiet areas without environmental stimuli [13].

Ten minutes was chosen as our time interval because it takes approximately ten minutes to reverse the medetomidine after administering intramuscular atipamezole [14].

Potential contraindications for medetomidine and atipamezole use include patients that may not tolerate sedation, such as dogs with cardiac disease, shock, respiratory disorders, certain liver and kidney diseases, or in dogs that are severely debilitated. Patient selection should be performed via a complete physical examination, CBC, serum chemistry, urinalysis, and blood pressure measurement. None of the patients in either phase of this study had any notable adverse effects of the pharmaceuticals.

\section{Conclusion}

We conclude that administration of low dose medetomidine (2 $\mu \mathrm{g} / \mathrm{kg}, \mathrm{IV})$ or dexmedetomidine (1 $\mu \mathrm{g} / \mathrm{kg}, \mathrm{IV})$ followed by atipamezole (10 $\mu \mathrm{g} / \mathrm{kg}$, IM) reliably reduces the impact of movement artifacts on EEG recordings in the dog. EEG waveforms are slightly slower following drug administration; however, this effect is predictable and therefore should have no significant effect on EEG interpretation. The main indication for the use of medetomidine and atipamezole is to obtain EEG tracings that are minimally affected by patient movement artifacts. The minimal side effects and potential complications with the use of these drugs suggest that this method may be suitable in all but a few selected cases. Since the completion of this study, the low dose medetomidine or dexmedetomidine administration has been used routinely for patients undergoing EEG studies at Mississippi State University. An added benefit of this method has been the lack of necessity of local intramuscular injections of lidocaine to prevent muscle artifacts. This advantage also reduces patient discomfort in performing routine EEG studies.

\section{Acknowledgements}

The authors would like to thank Dr. Robert Wills for his expertise and analysis of the statistical data and Dr. Andrew Mackin for his input with the preparation of this manuscript.

\section{REFERENCES}

[1] J. M. Ducoté and C. W. Dewey, "Neurodiagnostic,” In: C. W. Dewey, Ed., A Practical Guide to Canine and Feline Neurology, Blackwell Publishing, Ames, 2003, pp. 99178. http://dx.doi.org/10.1111/j.1467-2995.2005.00221.x

[2] P. A. March and W. W. Muir, "Bispectral Analysis of the Electroencephalogram: A Review of Its Development and Use in Anesthesia," Veterinary Anaesthesia and Analgesia, Vol. 32, No. 5, 2005, pp. 241-255.

[3] H. S. Jang, Y. S. Kwon, M. G. Lee, et al., "The Effect of Tiletamine/Zolazepam (Zoletile) Combination with Xylazine or Medetomidine on Electroencephalograms in Dogs,” The Journal of Veterinary Medical Science, Vol. 66, No. 5, 2004, pp. 501-507. http://dx.doi.org/10.1292/jvms.66.501

[4] D. Stenberg, "Physiological Role of Alpha 2-Adrenoceptors in the Regulation of Vigilance and Pain: Effect of Medetomidine,” Acta Veterinaria Scandinavica. Supplementum, Vol. 85, 1989, pp. 21-28.

[5] M. D. Lorenz and J. N. Kornegay, "Seizures, Narcolepsy and Cataplexy,” In: M. D. Lorenz and J. N. Kornegay, Eds., Handbook of Veterinary Neurology, 4th Edition, Saunders, St. Louis, 2004, pp. 323-339.

[6] R. W. Redding, R. B. Prynn and J. L. Wagner, "Clinical Use of the Electroencephalogram in Canine Encephalitis," American Veterinary Medical Association, Vol. 148, 1966, pp. 141-149.

[7] N. Lemo, D. Vnuk, B. Radisic, et al., "Determination of the Toxic Dose of Lidocaine in Dogs and Its Corresponding Serum Concentration,” Veterinary Record, Vol. 
160, No. 11, 2007, pp. 374-375.

http://dx.doi.org/10.1136/vr.160.11.374

[8] W. B. Pickworth, L. G. Sharpe and V. N. Gupta, "Morphine-Like Effects of Clonidine on the EEG, Slow Wave Sleep and Behavior in the Dog," European Journal of Pharmacology, Vol. 81, No. 4, 1982, pp. 551-557. http://dx.doi.org/10.1016/0014-2999(82)90344-2

[9] K. Itamoto, Y. Taura, N. Wada, et al., "Effect of Medetomidine on Electroencephalography and Use of a Quantitative Electroencephalograph for Evaluating Sedation Levels in Dogs,” Journal of Veterinary Medicine Series A, Vol. 48, No. 9, 2001, pp. 525-535. http://dx.doi.org/10.1046/j.1439-0442.2001.00381.x

[10] R. Virtanen, "Pharmacological Profiles of Medetomidine and Its Antagonist, Atipamezole," Acta Veterinaria Scandinavica, Vol. 85, 1989, pp. 29-37.

[11] A. Pitkänen, S. Narkilahti, Z. Bezvenyuk, et al., “Atipa- mezole, an Alpha(2)-Adrenoceptor Antagonist, Has Disease Modifying Effects on Epileptogenesis in Rats," Epilepsy Research, Vol. 61, No. 1-3, 2004, pp. 119-140. http://dx.doi.org/10.1016/j.eplepsyres.2004.07.005

[12] M. D. Sinclair, "A Review of the Physiological Effects of Alpha2-Agonists Related to the Clinical Use of Medetomidine in Small Animal Practice," The Canadian Veterinary Journal, Vol. 44, No. 11, 2003, pp. 885-897.

[13] C. E. Short, “Alpha 2 Agents in Animals,” In: C. E. Short, Ed., Alpha 2 Agents in Animals Sedation, Analgesia and Anaesthesia, Vol. 1, Veterinary Practice Publishing, Santa Barbara, 1992, pp. 1-55.

[14] O. Vainio and T. Vaha-Vahe, "Reversal of Medetomidine Sedation by Atipamezole in Dogs," Journal of Veterinary Pharmacology and Therapeutics, Vol. 13, 1990, pp. 1522. http://dx.doi.org/10.1111/j.1365-2885.1990.tb00742.x 